\title{
Optimizing Oxygen Reduction Catalyst Morphologies from First Principles
}

\author{
Ehsan A. Ahmad, ${ }^{* \dagger, \ddagger V}$ Vasiliki Tileli, Denis Kramer, ${ }^{\S}$ Giuseppe Mallia, ${ }^{\dagger, \ddagger}$ \\ Kelsey A. Stoerzinger," Yang Shao-Horn," Anthony R. Kucernak, ${ }^{\dagger}$ and \\ Nicholas M. Harrison ${ }^{\dagger, \ddagger}, \perp$
}

Department of Chemistry, Imperial College London, South Kensington, London SWY 2AZ, UK, Thomas Young Centre, Imperial College London, South Kensington, London SW' 2AZ, UK, Department of Materials, Imperial College London, South Kensington, London SW7 2AZ, UK, Faculty of Engineering and the Environment, University of Southampton, University Road, Southampton SO17 1BJ, UK, Electrochemical Energy Laboratory, Department of Mechanical Engineering, Massachusetts Institute of Technology, 77 Massachusetts Avenue, Cambridge, Massachusetts 02139, USA, and Daresbury Laboratory, Daresbury, Warrington, WA4 4AD, United Kingdom

E-mail: ea808@imperial.ac.uk

\footnotetext{
${ }^{*}$ To whom correspondence should be addressed

${ }^{\dagger}$ Department of Chemistry, Imperial College London, South Kensington, London SW7 2AZ, UK

¥Thomas Young Centre, Imperial College London, South Kensington, London SW7 2AZ, UK

"Department of Materials, Imperial College London, South Kensington, London SW7 2AZ, UK

${ }^{\S}$ Faculty of Engineering and the Environment, University of Southampton, University Road, Southampton SO17 1BJ, UK

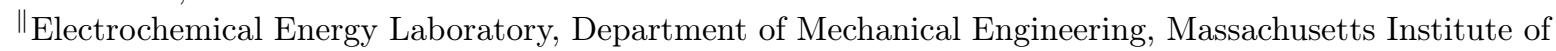
Technology, 77 Massachusetts Avenue, Cambridge, Massachusetts 02139, USA

${ }^{\perp}$ Daresbury Laboratory, Daresbury, Warrington, WA4 4AD, United Kingdom
} 


\begin{abstract}
Catalytic activity of perovskites for oxygen reduction (ORR) was recently correlated with bulk d-electron occupancy of the transition metal. We expand on the resultant model, which successfully reproduces the high activity of $\mathrm{LaMnO}_{3}$ relative to other perovskites, by addressing catalyst surface morphology as an important aspect of the optimal ORR catalyst. The nature of reaction sites on low index surfaces of orthorhombic (Pnma) $\mathrm{LaMnO}_{3}$ is established from First Principles. The adsorption of $\mathrm{O}_{2}$ is markedly influenced by local geometry and strong electron correlation. Only one of the six reactions sites that result from experimentally confirmed symmetry-breaking Jahn-Teller distortions is found to bind $\mathrm{O}_{2}$ with an intermediate binding energy while facilitating the formation of superoxide, an important ORR intermediate in alkaline media. As demonstrated here for $\mathrm{LaMnO}_{3}$, rational design of the catalyst morphology to promote specific active sites is a highly effective optimization strategy for advanced functional ORR catalysts.
\end{abstract}

\title{
Keywords
}

American Chemical Society, Electrocatalysis, alkaline fuel cells, Perovskites, oxygen reduction reaction, high resolution transmission electron microscopy $\mathrm{AT}_{\mathrm{E} X} \mathrm{X}$

\section{Introduction}

Perovskites have been studied for their activity towards the oxygen reduction reaction (ORR) since the 1970s. ${ }^{1-3}$ Interest in alkaline fuel cells (AFCs) has been renewed in the last decade because of the development of suitable hydroxide-exchange membrane materials allowing the alkaline equivalent of the Proton Exchange Membrane Fuel Cell (PEMFC). ${ }^{4}$ In AFC systems low-cost transition metals (TMs) are both stable and catalyse the ORR well, in contrast to the acidic PEMFC where only expensive noble metal catalysts show suitable 
activity and stability in spite of decades of research. ${ }^{5,6} \mathrm{LaMnO}_{3}$ has been identified to be amongst the most catalytically active perovskites, and recent experiments on a range of perovskites suggest that the high performance is linked to the $d^{4}$ character of the $\mathrm{Mn}^{3+}$ ion. This has been attributed to the single $\mathrm{Mn} e_{g}$ electron allowing for an interaction with $\mathrm{O}_{2}$ molecules that is neither too strong nor too weak. ${ }^{7,8}$

The strength of the TM-O $\mathrm{O}_{2}$ interaction has a significant effect on the activity of perovskite catalysts for the ORR. Previous analysis of the reaction mechanism has focused on the bulk electronic structure and simple models based on the covalent interaction of metals ions with adsorbed species. These models do not give full consideration to the important role of electron correlation in generating strong Jahn-Teller distortions of the $\mathrm{MO}_{6}$ octahedra in the active $d^{4}$ (and $\left.d^{7}\right)$ perovskites. This gives rise to a symmetry breaking orbital ordering of the bulk crystal and, as will be shown below, significant variations in surface reaction sites.

There have been previous studies of the adsorption of oxygen on the surface of $\mathrm{LaMnO}_{3},{ }^{9-11}$ but the majority of studies have focussed on solid oxide fuel cell (SOFC) applications and therefore consider only the highly symmetric, high temperature, cubic phase. For the lower operating temperature of AFCs, it is the orbitally ordered orthorhombic phase that is relevant. ${ }^{12-14}$ The schematic in Figure 1 shows that the orthorhombic distortion of the low temperature phase leads to a set of crystallographically distinct surfaces, which are likely to exhibit different reactivities. The manufacturing procedure and resulting surface terminations have also shown to play a crucial role in determining catalytic activity. ${ }^{15}$ We use first principles calculations, which reproduce the effects of strong electronic correlation, to demonstrate that local Jahn-Teller (J-T) distortions play an important role in determining the interaction of $\mathrm{O}_{2}$ molecules with $\mathrm{LaMnO}_{3}$ surfaces. The insight gained is of direct relevance to the design of new catalysts.

Previous studies of $\mathrm{O}_{2}$ adsorbtion on orthorhombic (Pnma) $\mathrm{LaMnO}_{3}$ have considered the (010) and (001) surfaces. ${ }^{10,16,17}$ The studies focus on a range of adsorption modes and binding sites, again with consideration to the SOFC reaction mechanism. ${ }^{10,16}$ Among the 
likely reaction sites in an AFC environment, only the five coordinated Mn octaherdon $\left(\mathrm{MnO}_{5}\right)$ at the (001) surface has so far been the subject of first principles investigations. ${ }^{17}$

None of the aforementioned studies specify whether the adsorption sites are J-T distorted, while our previous work ${ }^{14}$ indicates that a significant local distortion produces Mn-O contacts that are either elongated (apical 2.31 $\AA$ ) or shortened (equatorial 1.92/1.99 $\AA$ ) relative to the undistorted octahedra that prevail in the cubic high temperature phase. As the $\mathrm{O}_{2}$ binding energy is very sensitive to these local distortions, the use of a theory that takes into account strong on-site correlation is essential. This is severely underestimated in studies that use local or semi-local approximations (LDA or GGA) to density functional theory (DFT).

As the nature of $\mathrm{O}_{2}$ adsorption on the surfaces of orthorhombic $\mathrm{LaMnO}_{3}$ has yet to be

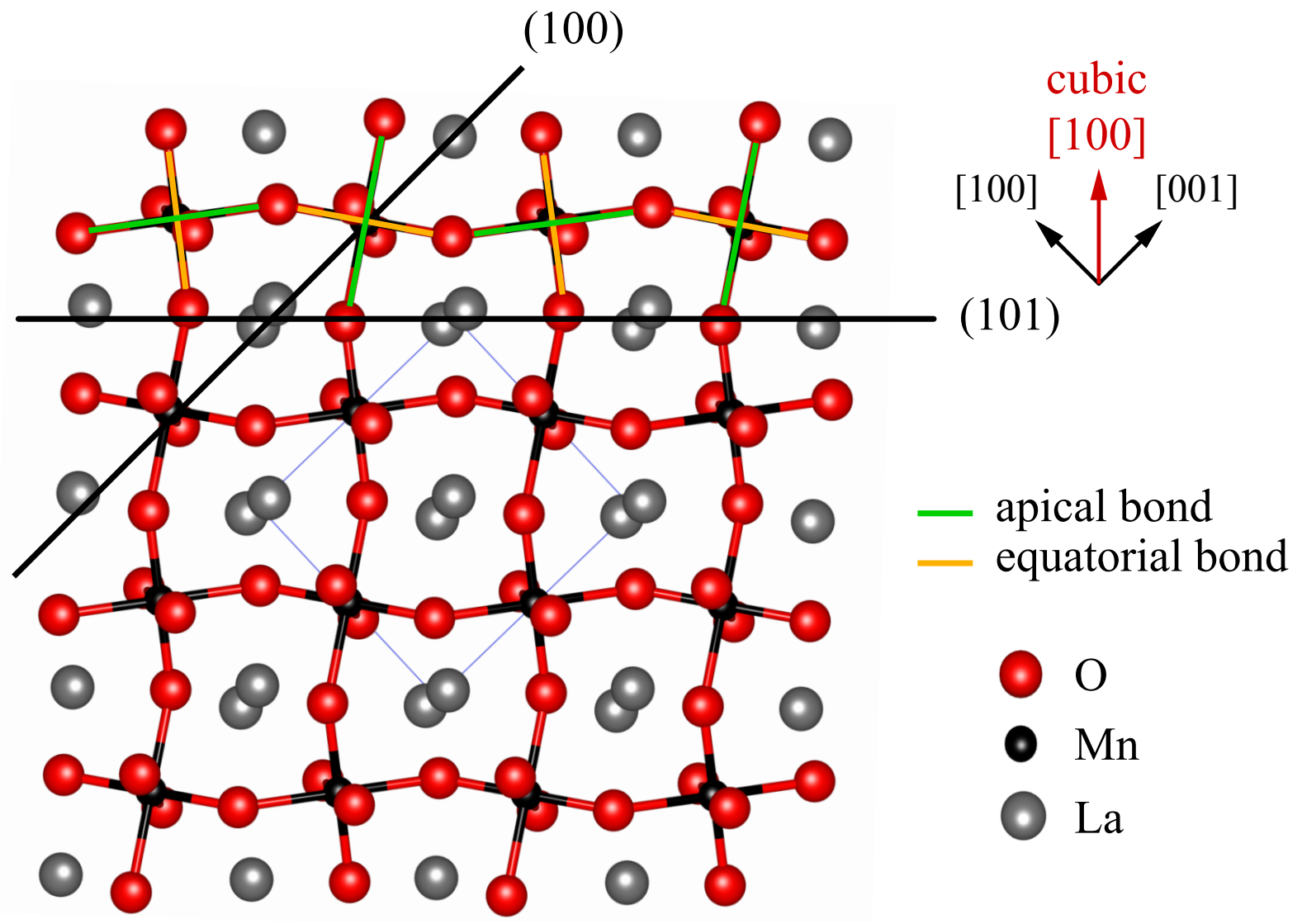

Figure 1: Crystal structure of orthorhombic $\mathrm{LaMnO}_{3}$; the bulk unit cell is given in blue and cleavage lines in black. Note that the [100] direction of the cubic phase corresponds to [101] in the standard orthorhombic setting (Pnma). 
fully explored, we provide a systematic characterisation of the binding interaction of $\mathrm{O}_{2}$ on the full range of sites available on the non-polar, stoichiometric and unreconstructed lowindex surfaces, established in our previous work. ${ }^{18}$ The six distinct types of adsorption site are listed in Table 1 and characterised by the surface on which they occur, the number of $\mathrm{O}$ atoms cleaved to form the site, and the J-T distortion of the Mn-O bond cleaved when forming the site. These sites are indicative of those present in an active catalyst under reaction conditions as they provide examples of apically and equatorially distorted 4- and 5-coordinate Mn sites. The strength of $\mathrm{O}_{2}$ binding is relatively easily computed and is likely to be an excellent proxy for ORR activity as the formation of reduced $\mathrm{O}_{2}$ at the surface is known to be an important intermediate in the high $\mathrm{pH}$ environment of the AFC. ${ }^{19-21}$ The use of binding energies to determine reactivity has been exploited in previous studies where a middle ground is sought between overly strong or weak binding of adsorbates for higher catalytic activity. ${ }^{7,8}$ We also use the charge transfer to the adsorbed molecule as an indication of the likelihood of reaction steps that depend on the oxidation of the metal site, and therefore assert that the combination of binding energy and charge transfer provide a strong indication of the catalytic activity of the sites considered.

Table 1: The TM adsorption sites available in each primitive unit cell of the non-polar, stoichiometric and unreconstructed low-index surfaces of $\mathrm{LaMnO}_{3}$ (given in order of increasing surface formation energies $\left(\mathbf{E}_{s}\right)$. The sites are $\mathrm{J}-\mathrm{T}$ distorted octahedra $\left(\mathrm{MnO}_{6}\right)$ from which $\mathrm{O}$ ions have been cleaved $\left(\mathrm{MnO}_{5}\right.$ or $\mathrm{MnO}_{4}$ ) in the formation of the surface. The superscripts $A p$ (apical) and $E q$ (equatorial) indicate the type of $\mathrm{Mn}-\mathrm{O}$ bond cleaved from the octahedron to form the site. ${ }^{18}$

\begin{tabular}{ccc}
\hline Plane & $\mathrm{E}_{s}$ Relaxed $\left(\mathrm{J} / \mathrm{m}^{2}\right)$ & Mn Sites \\
\hline$(100)$ & 0.98 & $2 \mathrm{MnO}_{5}^{A p}$ \\
$(001)$ & 1.39 & $2 \mathrm{MnO}_{5}^{E q}$ \\
$(101)$ & 1.36 & $2 \mathrm{MnO}_{5}^{A p}$ \\
$(110)$ & 1.98 & $1 \mathrm{MnO}_{5}^{A p} 1 \mathrm{MnO}_{4}^{A p+E q}$ \\
\hline
\end{tabular}




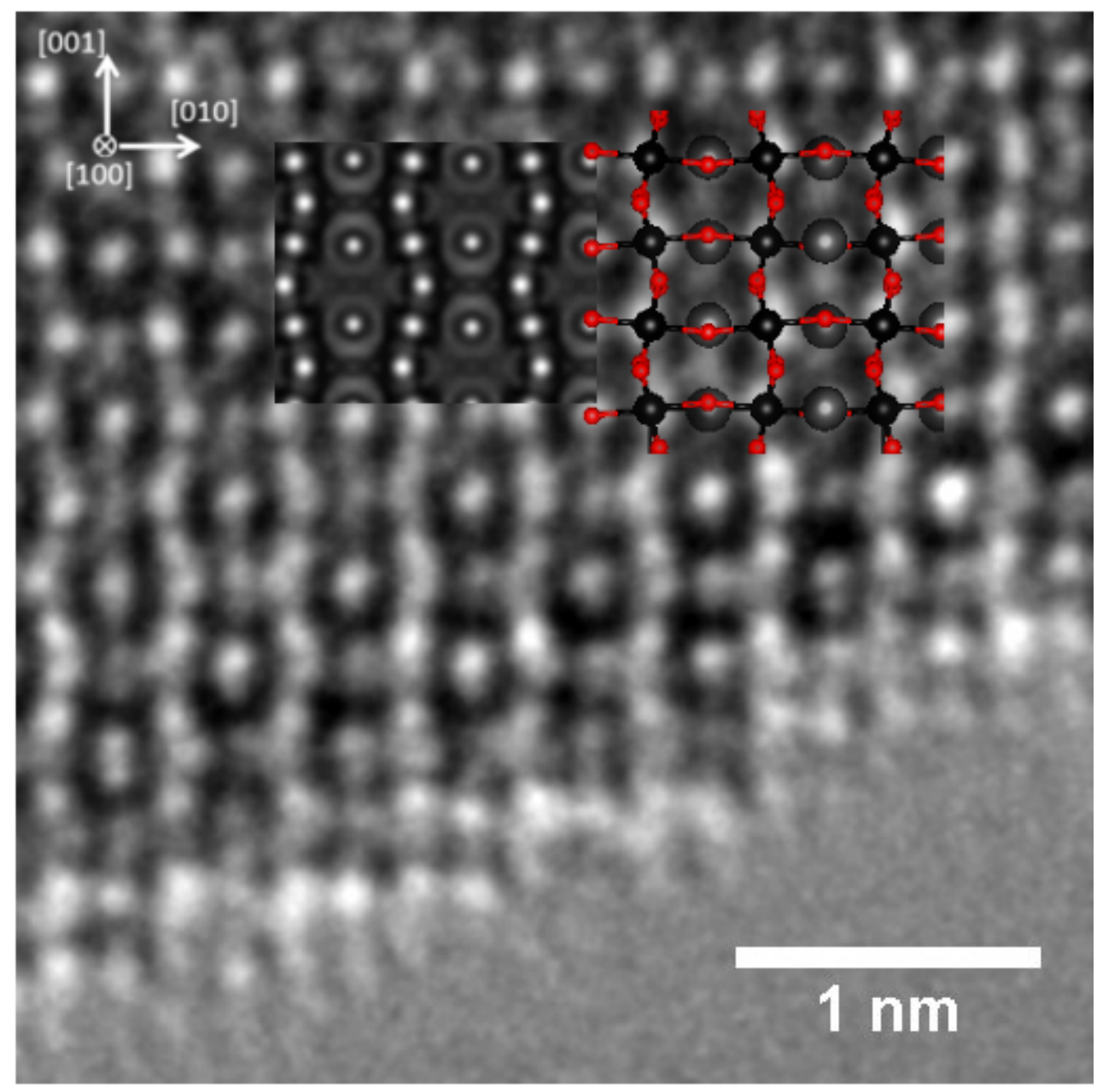

Figure 2: TEM image of a $\mathrm{LaMnO}_{3}$ particle edge showing the Jahn-Teller distortion at the exposed surface of the (001) plane. In the overlaid schematics, a simulated HRTEM image is shown along with simulations of the atomic positions where La is grey, $\mathrm{Mn}$ is black, and $\mathrm{O}$ is red.

\section{Results}

To verify the existence of J-T distortion at the orthorhombic $\mathrm{LaMnO}_{3}$ surface, TEM was performed on crystalline powder. A typical image is shown in Figure 2 in which the predicted J-T distortion of Mn-O octahedra in the bulk crystal is clearly visible and is perpetuated from the bulk to the surface. Heuristically, one would be inclined to propose a model for adsorption of $\mathrm{O}_{2}$ on these distorted surfaces where binding is stronger at lower coordinated $\mathrm{Mn}$ sites and at the shorter Mn-O bond provided by equatorial sites relative to the apical sites. 
While the former is confirmed by our detailed study, the complex nature of the interaction between $\mathrm{O}_{2}$ and the active sites leads to a non-intuitive dependence of adsorption strength with bond length.

The computed electronic and structural data for the adsorption of $\mathrm{O}_{2}$ at each site is presented together with binding energies in Table 2. Note that in the DFT calculations of the bulk-like regions of the $\mathrm{LaMnO}_{3}$ lattice, the charge of an $\mathrm{O}$ ion estimated by Mulliken population analysis (MPA) is $-1.3|e|$. Since $\mathrm{O}^{2-}$ is the formal oxidation state in bulk $\mathrm{LaMnO}_{3}$, a value of $-0.65|e|$ for the adsorbed molecule $\left(\mathrm{O}_{2} q\right.$ in Table 2) indicates a formal charge of $-1|e|$. The computed occupations of the surface Mn-3d orbitals are documented in Table 3. These reflect changes in the oxidation state of the surface sites.

Table 2: The computed binding energies $\mathrm{BE}$ and bond distances $\left(d\right.$, in $\AA$ ) of $\mathrm{O}_{2}$ adsorption on the $\mathrm{LaMnO}_{3}$ surfaces, indicating the initial > final adsorption site where different. $q\left(\mathrm{O}_{2}\right)$ is the charge transferred in $|e|$ to $\mathrm{O}_{2}$ and resulting species. (where $\mathbf{0 . 6 5}|e|$ by MPA is equivalent to 1 electron based on the bulk charges). $e_{g}$ refers to the formal $e_{g}$ electrons at the active site before $\mathrm{O}_{2}$ adsorption.

\begin{tabular}{lccccc}
\hline Adsorption Mode & $d\left({\left.\mathrm{Mn}-\mathrm{O}_{2}\right)}\right.$ & $d(\mathrm{O}-\mathrm{O})$ & $\mathrm{BE}(\mathrm{eV})$ & $q\left(\mathrm{O}_{2}\right)$ & $e_{g}$ \\
\hline Free $\mathrm{O}_{2}$ & - & 1.23 & - & 0 & \\
$(100) \mathrm{MnO}_{5}^{A p}$ & 2.00 & 1.31 & -0.15 & $0.48\left(\mathrm{O}_{-} \mathrm{O}^{-}\right)$ & 1 \\
$(001) \mathrm{MnO}_{5}^{E q}$ & 2.07 & 1.34 & -0.61 & $0.61\left(\mathrm{O}-\mathrm{O}^{-}\right)$ & 1 \\
$(101) \mathrm{MnO}_{5}^{A p}>\mathrm{La}$ bridge & - & 1.36 & -1.17 & $0.74\left(\mathrm{O}_{-} \mathrm{O}^{-}\right)$ & - \\
$(110) \mathrm{MnO}_{5}^{A p}>\mathrm{MnO}_{4}^{E q-\text { Lateral }}$ & 1.93 & 1.48 & -2.04 & $1.24\left(\mathrm{O}_{-} \mathrm{O}^{2-}\right)$ & 2 \\
$(110) \mathrm{MnO}_{4}^{A p}>\mathrm{MnO}_{4}^{E q-E n d-o n}$ & 2.03 & 1.34 & -2.32 & $0.70\left(\mathrm{O}_{-} \mathrm{O}^{-}\right)$ & 2 \\
$(110) \mathrm{MnO}_{4}^{E q}>\mathrm{MnO}_{4}^{E q-\text { Bidentate }}$ & 1.94 & 1.46 & -2.67 & $1.04\left(\mathrm{O}-\mathrm{O}^{2-}\right)$ & 2 \\
\hline
\end{tabular}

It is evident that $\mathrm{O}_{2}$ adsorption to $\mathrm{MnO}_{5}$ sites results in strong charge transfer to the molecule and oxidation of $\mathrm{Mn}$ from $d^{4}$ to $d^{3}$. This is accompanied by a large surface relaxation that may be interpreted as an anti-J-T distortion as the $e_{g}$ orbital is no longer occupied.

Table 3: The occupations of the Mn-3 $d$ orbitals of the adsorption sites estimated by MPA before and after $\mathrm{O}_{2}$ adsorption.

\begin{tabular}{lcc}
\hline Adsorption Mode & Initial $3 d(|e|)$ & Final $3 d(|e|)$ \\
\hline$(100) \mathrm{MnO}_{5}^{A p}$ & 3.75 & 3.06 \\
$(001) \mathrm{MnO}_{5}^{E q}$ & 3.75 & 2.91 \\
$(101) \mathrm{MnO}_{5}^{A p}>$ La bridge & - & - \\
$(110) \mathrm{MnO}_{5}^{A p}>\mathrm{MnO}_{4}^{E q-\text { Lateral }}$ & $3.69,4.52$ & $2.70,3.77$ \\
$(110) \mathrm{MnO}_{4}^{A p}>\mathrm{MnO}_{4}^{E q-E n d-o n}$ & $3.69,4.52$ & $3.21,3.70$ \\
$(110) \mathrm{MnO}_{4}^{E q}>\mathrm{MnO}_{4}^{E q-\text { Bidentate }}$ & $3.69,4.52$ & $3.17,2.99$ \\
\hline
\end{tabular}


This is illustrated very clearly in the spin density at the surface sites before and after $\mathrm{O}_{2}$ adsorption (Figure 3). The final geometry of each $\mathrm{MnO}_{5}$ site is therefore rather similar, and the critical role that the apical and equatorial bond lengths of the initial geometry play in the adsorption energetics is not reflected in the final $\mathrm{Mn}-\mathrm{O}_{2}$ distances. This results in a rather unexpected trend in adsorption energy with $\mathrm{Mn}-\mathrm{O}_{2}$ distance, as typically one would expect stronger binding to be correlated with a shorter interatomic distance.

The differences in the initial coordination of the reaction sites, on the other hand, are reflected in the final $\mathrm{Mn}-\mathrm{O}_{2}$ distances. $\mathrm{O}_{2}$ adsorption on the $\mathrm{MnO}_{4}$ sites results in significantly lower $d\left(\mathrm{Mn}-\mathrm{O}_{2}\right)$, as listed in Table 2. The End - on adsorption mode on the $\mathrm{MnO}_{4}$ site proves to be an exception to this, as in this mode the adsorption results in formation of a superoxide $\left(\mathrm{O}_{2}{ }^{-}\right)$rather than the peroxide $\left(\mathrm{O}_{2}{ }^{2-}\right)$. In the following we analyze the nature of the adsorption at each of the six surface reaction sites (Figures 4 - 7) in detail.

(100) $\mathrm{MnO}_{5}^{A p}$ The lowest binding energy and charge transfer $(-0.15 \mathrm{eV}$ and $0.48|e|)$ are observed for $\mathrm{O}_{2}$ adsorption on this site. Adsorption takes place at an elongated 5 coordinate apical site and the $d(\mathrm{O}-\mathrm{O})$ of $1.31 \AA$ is the least perturbed from that computed for the free $\mathrm{O}_{2}$ molecule. This is consistent with a general trend that $d(\mathrm{O}-\mathrm{O})$ increases in proportion to the binding energy. ${ }^{9,11,22}$

(001) $\mathrm{MnO}_{5}^{E q}$ This site is similar to that on the (100) surface but is formed from the cleavage of an equatorial bond. Adsorption is therefore far stronger with a binding energy of $-0.61 \mathrm{eV}$, with significantly higher charge transfer and, as expected, a somewhat longer $d(\mathrm{O}-\mathrm{O})$ of $1.34 \AA$.

(101) $\mathrm{MnO}_{5}^{A p}>$ La bridge The geometry of the (101) surface results in a unique adsorption mode for the $\mathrm{O}_{2}$ molecule where it is not bound to the $\mathrm{MnO}_{5}^{A p}$ site. Instead, as shown in Figure 6, the minimum energy geometry is in a bridging position between the surface La 
a)

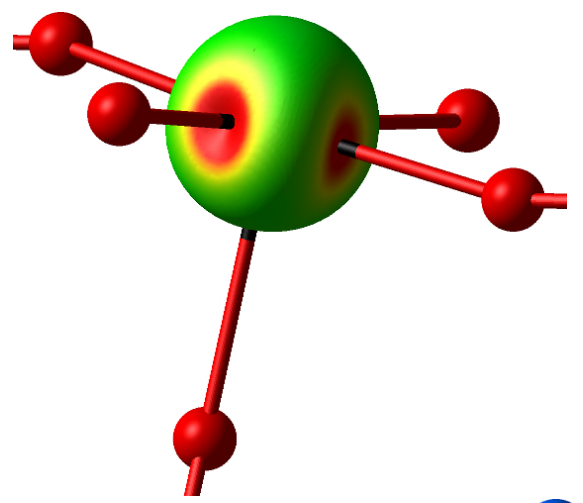

b)

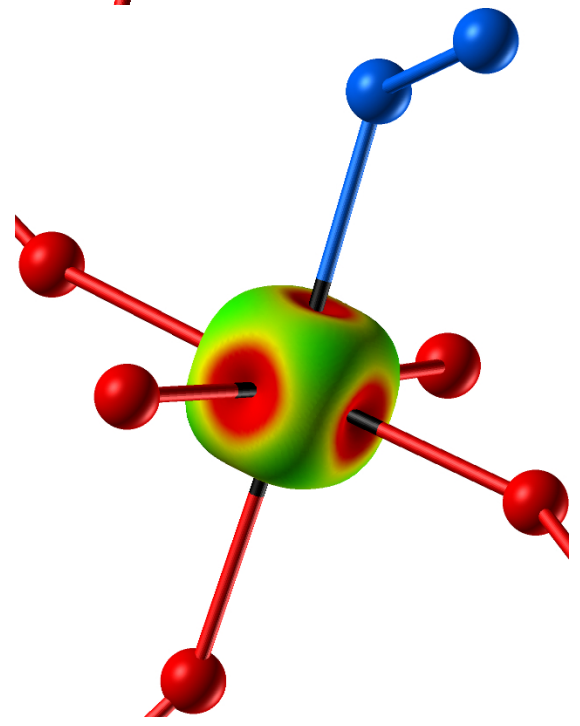

c)

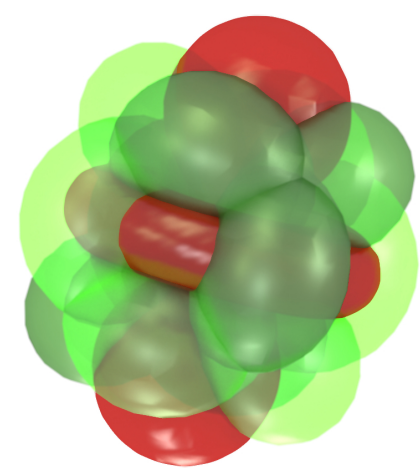

Figure 3: The calculated spin density of $a$ ) the unoccupied $\mathrm{Mn}_{5}^{A p}$ site on the (100) surface, and $b$ ) after $\mathrm{O}_{2}$ adsorption. $c$ ) shows a model of the idealized $d_{x y}, d_{x z}, d_{y z}$ and $d_{z^{2}}$ suborbitals for reference.

ions. The binding energy at this site is $-1.17 \mathrm{eV}$ compared to the -0.15 we observe for the $\mathrm{MnO}_{5}^{A p}$ site. The higher binding energy and charge transfer $(-0.74|e|)$ are reflected 


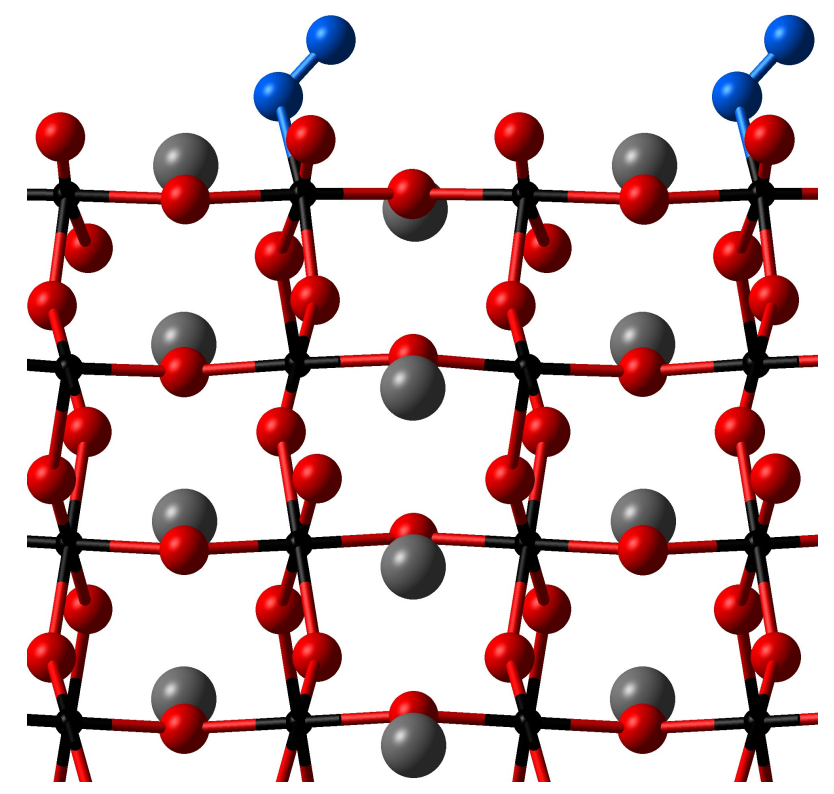

Figure 4: Optimised geometry of the (100) surface with $\mathrm{O}_{2}$ adsorbed on the $\mathrm{MnO}_{5}^{A p}$ site.

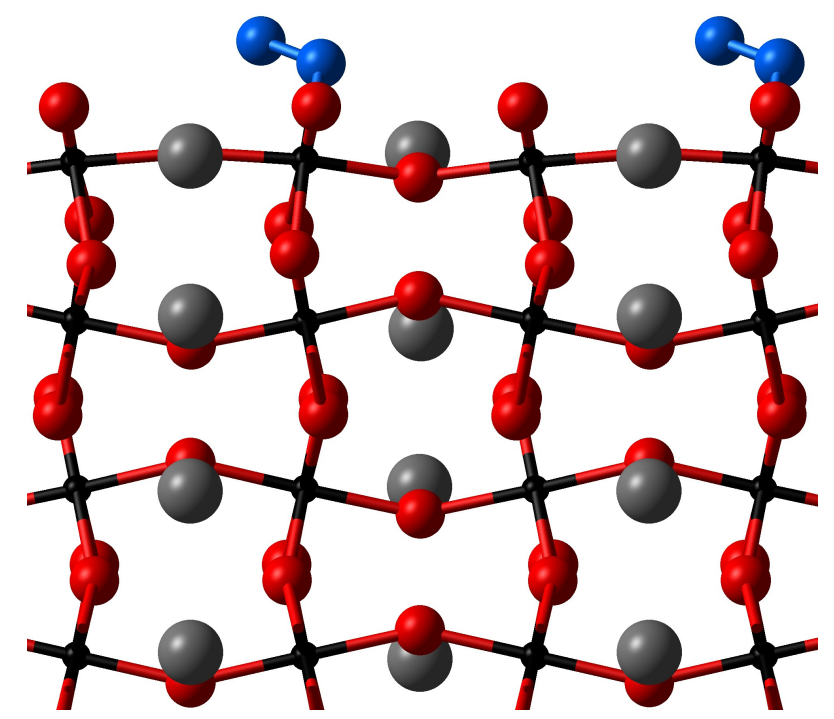

Figure 5: Optimised geometry of the (001) surface with $\mathrm{O}_{2}$ adsorbed on the $\mathrm{MnO}_{5}^{E q}$ site.

in the longer $d(\mathrm{O}-\mathrm{O})$ of $1.36 \AA$. In this case the charge is transferred from fully coordinate $\mathrm{MnO}_{6}$ octahedra at the surface rather than the surface $\mathrm{MnO}_{5}$ sites (Figure 6, charge transfer quantified in Supporting Table S2). The adsorption geometry and charge transfer here suggest an alternative pathway for the ORR, via electron transfer from the surface rather than from a specific site, which has not been discussed in previous literature. Studies of $\mathrm{O}_{2}$ 
adsorption on the La site have only been carried out for the high temperature cubic $\mathrm{LaMnO}_{3}$ surfaces, where it is reported that binding is generally weaker than at Mn sites. ${ }^{9}$ The (101) surface results in this adsorption mode as access to the Mn site in the second surface layer is sterically inhibited and the surface layer contains two La ions in close proximity (Figure 6).

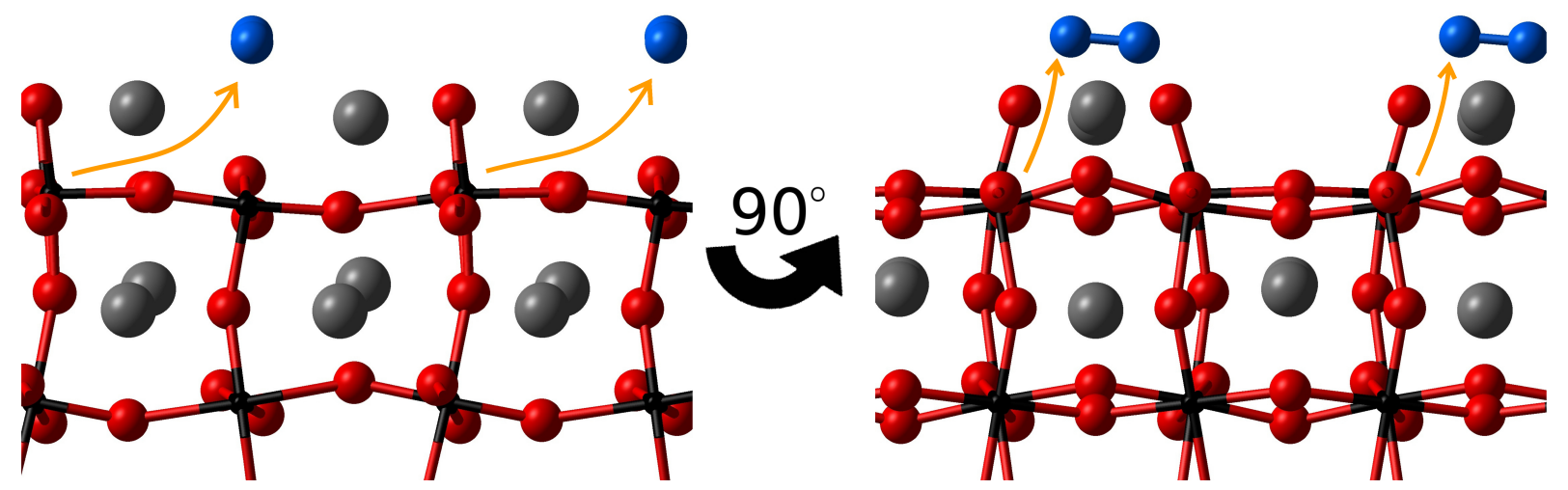

Figure 6: Optimised geometry of the (101) surface with $\mathrm{O}_{2}$ adsorbed on the La bridge site. Rotated view of the cell is included to clarify $\mathrm{O}_{2}$ adsorption geometry. Orange arrows indicate charge donating surface Mn.

(110) This surface presents three distinct types of adsorption site: $\mathrm{MnO}_{5}^{A p}, \mathrm{MnO}_{4}^{A p}$ and $\mathrm{MnO}_{4}^{E q}$. We investigated these sites independently by adsorbing an $\mathrm{O}_{2}$ molecule at an initial geometry above the sites. However, as observed in Figure 7, three meta-stable adsorption modes are obtained that centre on the $\mathrm{MnO}_{4}^{E q}$ site: $\mathrm{MnO}_{4}^{\text {Eq-Lateral }}, \mathrm{MnO}_{4}^{\text {Eq-End-on }}$ and $\mathrm{MnO}_{4}^{E q-\text { Bidentate }}$, resulting in binding energies of $-2.04,-2.32$ and $-2.67 \mathrm{eV}$ respectively. The $d(\mathrm{O}-\mathrm{O})$, which has been linear with binding energy for the other surfaces, appears to be insensitive to the binding energies on the $\mathrm{MnO}_{4}^{E q}$ site. The trend in $d(\mathrm{O}-\mathrm{O})$ can instead be rationalised more accurately by the variations in charge transfer, since increased filling of the antibonding $\mathrm{O}_{2} \pi^{*}$ orbital destabilises the $\mathrm{O}-\mathrm{O}$ bond. The $\mathrm{O}_{2}$ molecules in the $\mathrm{MnO}_{4}^{\text {Eq-Lateral }}$ and $\mathrm{MnO}_{4}^{E q-B i d e n t a t e}$ modes accept -1.24 $|e|$ and -1.04 $|e|$ while in the $\mathrm{MnO}_{4}^{E q-E n d-o n}$ mode it accepts $-0.70|e|$, resulting in $d(\mathrm{O}-\mathrm{O})$ of $1.48 \AA, 1.46 \AA$ and $1.34 \AA$ respectively.

The differences in binding energy between the adsorption modes on the $\mathrm{MnO}_{4}^{E q}$ site occur in part due to variations in structural relaxation stemming from the orientation of the $\mathrm{O}_{2}$ 

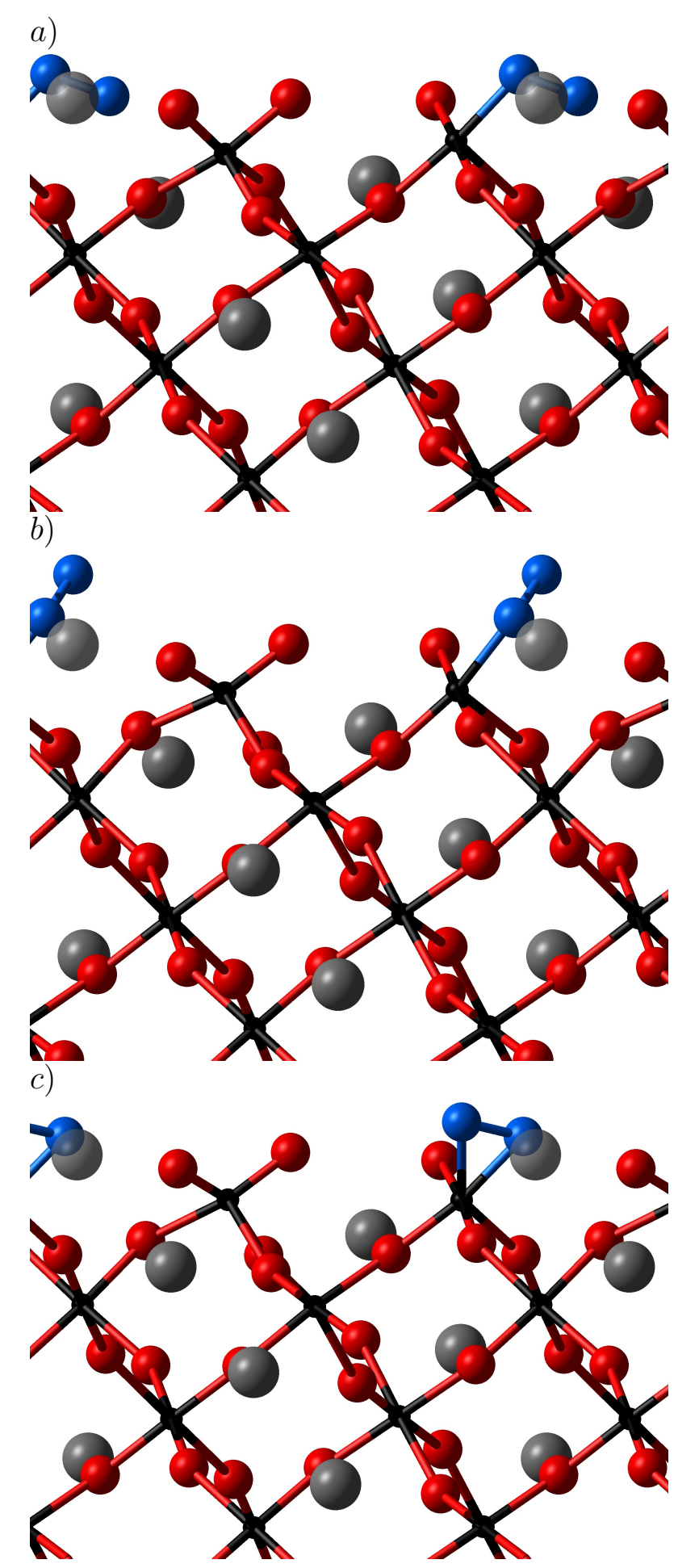

Figure 7: The (110) surface showing the adsorption modes: a) $\mathrm{MnO}_{4}^{\text {Eq-Lateral }}$ b) $\mathrm{MnO}_{4}^{E q-E n d-o n}$ c) $\mathrm{MnO}_{4}^{E q-B i d e n t a t e}$. The corresponding initial adsorption modes are provided in Table 2. 
molecule. End - on adsorption is known to be more energetically favourable than Lateral adsorption, perhaps due to lower interference with lattice O ions. ${ }^{10}$ For the $\mathrm{MnO}_{4}^{\text {Eq-Bidentate }}$ mode, the high binding energy can mostly be attributed to the stabilisation of both adsorption sites of the $\mathrm{MnO}_{4}$ octahedron simulataneously, which effectively cancels out its undercoordination and massively stabilises the surface.

Binding on the $\mathrm{MnO}_{4}^{E q}$ site of the (110) surface results in shorter $d\left(\mathrm{Mn}-\mathrm{O}_{2}\right)$ and significantly larger binding energies than observed on the $\mathrm{MnO}_{5}^{E q}$ of the (001) surface. This can be attributed to undercoordination. The $\mathrm{MnO}_{4}^{E q}$ site goes from 4 to 5 coordination upon $\mathrm{O}_{2}$ adsorption but still does not achieve the 6 coordination of the bulk, thus retains a stronger attraction between the $\mathrm{Mn}$ centre and the $\mathrm{O}_{2}$ molecule than observed on a $\mathrm{MnO}_{5}^{E q}$ site after $\mathrm{O}_{2}$ adsorption.

\section{Discussion}

Our current understanding of optimal ORR activity in $d^{4}$ and $d^{7}$ perovskites is based on the experimental study of a wide range of perovskites. ${ }^{7}$ The data suggests that the requirement for the ORR mechanism to proceed optimally is the existence of a single $e_{g}$ electron in the TM-ion, that is able to form a covalent interaction with the adsorbate. As a consequence, variations in the ORR kinetics between manganates $\left(d^{4}\right)$ and nickelates $\left(d^{7}\right)$ for instance, are seen to be a result of the changes in TM-O hybridization that modify the covalency of the TM-O ${ }_{2}$ (site-adsorbate) bond. However, this simplified concept of a single $e_{g}$ electron site and a covalent interaction is insufficient to describe the data presented herein. For example, the $\mathrm{Mn}_{5}^{A p}$ site that shows single $e_{g}$ occupancy produces weak charge transfer and binding when compared to the doubly $e_{g}$ occupied $\mathrm{MnO}_{4}^{E q}$ site, which generates strong charge transfer and binding.

After consideration of the computed binding energies and charge transfer at the various sites, we propose that the ORR mainly proceeds via the disproportionation reaction of 
adsorbed superoxide $\mathrm{O}_{2}^{-}$with water to form the readily desorbed hydroperoxide $\mathrm{HOO}^{-}$and hydroxide $\mathrm{OH}^{-} .6,20,21$ This pathway only proceeds via the mediation of catalyst sites that lead to the formation of $\mathrm{O}_{2}^{-}$. The sites which result in the formation of strongly bound peroxide $\mathrm{O}_{2}^{2-}$ are likely to give slower $\mathrm{OH}^{-}$formation kinetics due to the increased activation energy required to overcome the stronger $\mathrm{Mn}_{-} \mathrm{O}_{2}^{2-}$ bond. Optimal ORR activity is thus determined by two factors: firstly, the adsorption of $\mathrm{O}_{2}$ must be neither too weak nor too strong and, secondly, the charge transfer must result in the formation of superoxide that will react to form hydroperoxide. A typical sample of $\mathrm{LaMnO}_{3}$ will present a range of surface $\mathrm{Mn}$ sites that can form the superoxide species but due to the strong distortions of the truncated surface octahedra, and the variations in site coordination upon cleavage, only one of these sites is found to be particularly suitable for the ORR: the $\mathrm{MnO}_{5}^{E q}$ site on the (001) surface. At all other sites the binding energy is either too weak $\left(\mathrm{MnO}_{5}^{A p}\right.$ sites $)$ or too strong $\left(\mathrm{MnO}_{4}^{A p / E q}\right.$ sites).

The predicted relative inactivity of many sites on the catalyst may seem to be a strong contraindication for its use in alkaline fuel cells as only $20 \%$ of the catalyst surface is predicted to be the (001) surface. ${ }^{18}$ This result does, however, suggest a route to optimisation of the catalyst through control of the crystallite morphology to maximise the area of the (001) facets. As the data presented makes it clear that the binding energy of $\mathrm{O}_{2}$ is strongly affected by the distortion of the surface $\mathrm{MnO}_{5}$ sites, we further propose optimisation routes that vary the Mn-O distances of the surface sites. This includes doping in nano crystalline powders or strain engineering in epitaxial thin films. These strategies are highly likely to be succesful in catalyst optimsation and their implementation has been shown to be acheivable in recent experiments on $\mathrm{LaMnO}_{3}$ thin films. ${ }^{23}$ 


\section{Conclusions}

This work details the structure and energetics of the adsorption sites on the (100), (001), (101) and (110) surfaces of orthorhombic (Pnma) $\mathrm{LaMnO}_{3}$ by adsorption of molecular oxygen $\left(\mathrm{O}_{2}\right)$ in hybrid-exchange (B3LYP) density functional theory calculations. The binding energies of $\mathrm{O}_{2}$ indicate that $\mathrm{MnO}_{5}-d^{4}$ adsorption sites formed through cleavage of $\mathrm{J}-\mathrm{T}$ distorted apical bonds have a weak interaction with $\mathrm{O}_{2}$ while equatorially cleaved sites bind more strongly. In both instances, $\mathrm{O}_{2}$ is reduced to superoxide $\mathrm{O}_{2}^{-}$. Lower coordination sites $\left(\mathrm{MnO}_{4}-d^{5}\right)$ have overly strong binding and allow for the formation of peroxide $\mathrm{O}_{2}^{2-}$, which is not likely to be effective for the ORR in alkaline fuel cells (AFCs).

Only the $\mathrm{MnO}_{5}^{E q}$ site of the (001) surface satisfies the conditions of binding $\mathrm{O}_{2}$ with an energy neither too work nor too strong and results in the formation of a superoxide species necessary to proceed with the oxygen reduction reaction (ORR) in AFCs. This is shown to be due to the local distortion of the site which is caused by the J-T distortion of bulk $\mathrm{MnO}_{6}$ octahedra. This model suggests that manipulation of the site activity could be achieved by controlling bulk Mn-O distances through doping and lattice strain. In addition, the total activity can be increased by maximisation of active surface area (surfaces that exhibit $\mathrm{MnO}_{5}^{E q}$ sites) through control of the crystallite morphology. This work also establishes the need to consider the effects of strong on-site electron correlation in determining the geometry and chemical activity of reaction sites in transition metal oxide catalysts. It is therefore of direct relevance to the discovery and optimisation of functional transition metal oxides in many applications.

\section{Methodology}

Since previous studies have indicated that the ORR is catalysed via adsorption at a surface Mn site, ${ }^{7,20}$ we adopt an initial geometry for $\mathrm{O}_{2}$ adsorption with the molecule in an "end-on" orientation at the $\mathrm{Mn}$ site at a position reflecting the type of $\mathrm{Mn}-\mathrm{O}$ bond cleaved when 
forming the surface (Figure 8). Symmetric adsorption on both sides of a periodic slab is found to converge the adsorption energy rapidly as a function of slab thickness as it prevents the generation of an unphysical electrostatic dipole perpendicular to the surface. The predicted adsorption geometry is then generated by an unconstrained optimisation of all atomic coordinates. Details of the surface construction are provided in the supplementary information.

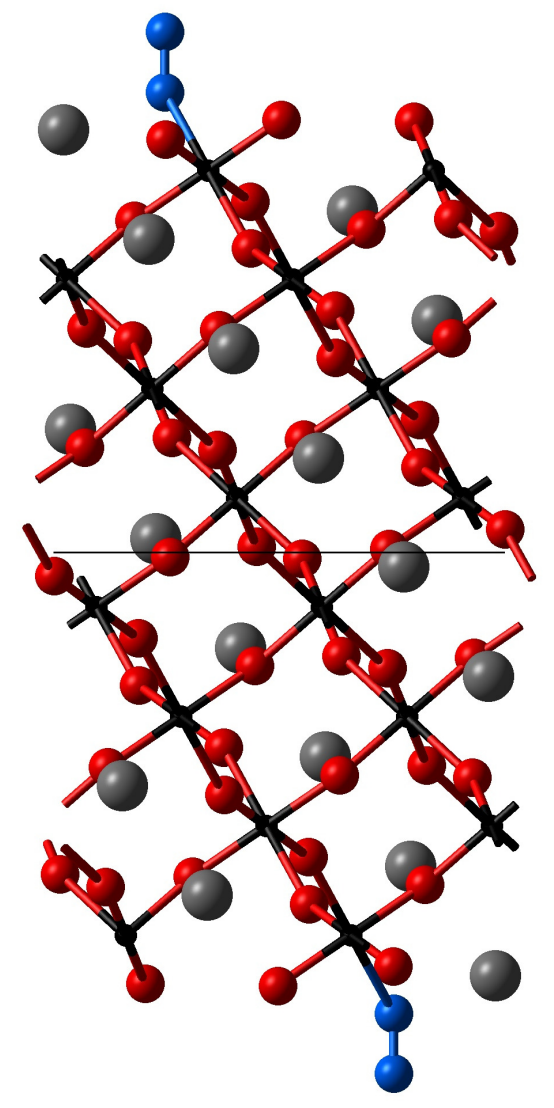

Figure 8: One unit cell of the (110) surface with an $\mathrm{O}_{2}$ molecule (blue) adsorbed on an apical Mn site $\left(\mathrm{MnO}_{5}^{A p}\right)$, before geometry optimisation. The lattice $\mathrm{Mn}, \mathrm{O}$ and La are represented by the small black, medium red and large grey spheres respectively.

Binding energies In general computed surface binding energies are corrected for basis set superposition error (BSSE) using a counterpoise correction (CPC). ${ }^{24-26}$ For systems studied in this work the $\mathrm{CPC}$ method is found to be inapplicable as strong charge rearrangements 
occur at the adsorption sites during relaxation. The BSSE is therefore estimated at the unrelaxed geometry. The details of this approach and the full list of binding energies obtained using the different methods of estimating BSSE is provided in the Supporting Information.

TEM The $\mathrm{LaMnO}_{3}$ single crystal particles were synthesized using a co-precipitation method as described elsewhere. ${ }^{7}$ High resolution transmission electron microscopy (TEM) was performed on a spherical aberration (Cs) corrected at the image plane FEI Titan 80-300 TEM operated at $300 \mathrm{kV}$. For enhancement of the contrast in the micrographs, the corrector was set at negative spherical aberration imaging conditions and it was tuned to $-13 \mu \mathrm{m} .{ }^{27}$ Simulations of the atomic positions to assist the interpretation of the high resolution micrographs were done using the multislice method in JEMS software. ${ }^{28}$

Computational Details We adopt the hybrid-exchange B3LYP functional for our DFT calculations, as it provides a description of strong on-site correlations and thus an accurate description of the energetics and electronic structure of systems in which strong electronelectron interactions result in electron localistion and orbital ordering (particularly for transition metal oxides). ${ }^{12,29-38}$ It has also recently been shown to provide quantitative agreement between calculated and experimental formation energies for a wide range of Mn-oxides including $\mathrm{LaMnO}_{3} \cdot{ }^{14}$

All calculations have been performed using the CRYSTAL09 ${ }^{39}$ code, based on the expansion of the crystalline orbitals as a linear combination of a local basis set (BS) consisting of atom centred Gaussian orbitals. The Mn and O ions are described by a triple valence allelectron BS: an 86-411d(41) contraction (one $s$, four $s p$, and two $d$ shells) and an 8-411d(1) contraction (one $s$, three $s p$, and one $d$ shells), respectively; the most diffuse $s p(d)$ exponents are $\alpha^{\mathrm{Mn}}=0.4986(0.249)$ and $\alpha^{\mathrm{O}}=0.1843(0.6) \mathrm{Bohr}^{-2} .{ }^{40}$ The La basis set includes a pseudopotential to describe the core electrons, while the valence part consists of a $411 \mathrm{p}(411) \mathrm{d}(311)$ contraction scheme (with three $s$, three $p$ and three $d$ shells); the most diffuse exponent is 
$\alpha^{\mathrm{La}}=0.15 \mathrm{Bohr}^{-2}$ for each $s, p$ and $d .^{12}$

The internal coordinates of the slab have been determined by minimization of the total energy within an iterative procedure based on the total energy gradient calculated with respect to the nuclear coordinates. Convergence was determined from the root-mean-square (rms) and the absolute value of the largest component of the forces. The thresholds for the maximum and the rms forces (the maximum and the rms atomic displacements) have been set to 0.00045 and 0.00030 (0.00180 and 0.0012) in atomic units. Geometry optimization was halted when all four conditions were satisfied simultaneously.

\section{Acknowledgement}

The authors thank Prof. Jin Suntivich for useful discussions. This work made use of the high performance computing facilities of Imperial College London and - via membership of the UK's HPC Materials Chemistry Consortium funded by EPSRC (EP/F067496) - of HECToR, the UK's national high-performance computing service, which is provided by UoE HPCx Ltd at the University of Edinburgh, Cray Inc and NAG Ltd, and funded by the Office of Science and Technology through EPSRC's High End Computing Programme.

\section{Supporting Information Available}

Procedure for binding energy calculations and the full Mn-3d orbital occupations of surface Mn ions. This material is available free of charge via the Internet at http://pubs.acs. org/.

\section{References}

(1) Tseung, A.; Bevan, H. A reversible oxygen electrode. Journal of Electroanalytical Chemistry and Interfacial Electrochemistry 1973, 45, 429 - 438. 
(2) Matumoto, Y.; Yoneyama, H.; Tamura, H. A new catalyst for cathodic reduction of oxygen: Lanthanum Nickel Oxide. Chemistry Letters 1975, 4, 661-662.

(3) Matsumoto, Y.; Yoneyama, H.; Tamura, H. Catalytic activity for electrochemical reduction of oxygen of lanthanum nickel oxide and related oxides. Journal of Electroanalytical Chemistry and Interfacial Electrochemistry 1977, 79, 319 - 326.

(4) Varcoe, J. R.; Atanassov, P.; Dekel, D. R.; Herring, A. M.; Hickner, M. A.; Kohl, P. A.; Kucernak, A. R.; Mustain, W. E.; Nijmeijer, K.; Scott, K. et al. Anion-exchange membranes in electrochemical energy systems. Energy Environ. Sci. 2014, 7, 3135-3191.

(5) Hayashi, M.; Uemura, H.; Shimanoe, K.; Miura, N.; Yamazoe, N. Reverse Micelle Assisted Dispersion of Lanthanum Manganite on Carbon Support for Oxygen Reduction Cathode. Journal of The Electrochemical Society 2004, 151, A158-A163.

(6) Bidault, F.; Brett, D.; Middleton, P.; Brandon, N. Review of gas diffusion cathodes for alkaline fuel cells. Journal of Power Sources 2009, 187, 39 - 48.

(7) Suntivich, J.; Gasteiger, H. A.; Yabuuchi, N.; Nakanishi, H.; Goodenough, J. B.; ShaoHorn, Y. Design principles for oxygen-reduction activity on perovskite oxide catalysts for fuel cells and metal - air batteries. Nature Chemistry 2011, 3, 546-550.

(8) Vojvodic, A.; Norskov, J. K. Optimizing Perovskites for the Water-Splitting Reaction. Science 2011, 334, 1355-1356.

(9) Choi, Y.; Mebane, D. S.; Lin, M. C.; Liu, M. Oxygen Reduction on LaMnO${ }_{3}$-Based Cathode Materials in Solid Oxide Fuel Cells. Chemistry of Materials 2007, 19, 16901699.

(10) Kotomin, E. A.; Mastrikov, Y.; Heifets, E.; Maier, J. Adsorption of atomic and molecular oxygen on the $\mathrm{LaMnO}_{3}(001)$ surface: ab initio supercell calculations and thermodynamics. Phys. Chem. Chem. Phys. 2008, 10, 4644-4649. 
(11) Choi, Y.; Lynch, M. E.; Lin, M. C.; Liu, M. Prediction of O2 Dissociation Kinetics on $\mathrm{LaMnO}_{3}$-Based Cathode Materials for Solid Oxide Fuel Cells. The Journal of Physical Chemistry C 2009, 113, 7290-7297.

(12) Muñoz, D.; Harrison, N. M.; Illas, F. Electronic and magnetic structure of $\mathrm{LaMnO}_{3}$ from hybrid periodic density-functional theory. Phys. Rev. B 2004, 69, 085115.

(13) Goodenough, J. B. .; Zhou, J.-S. Orbital ordering in orthorhombic perovskites. Journal of Materials Chemistry 2007, 17, 2394-2405.

(14) Ahmad, E. A.; Liborio, L.; Kramer, D.; Mallia, G.; Kucernak, A. R.; Harrison, N. M. Thermodynamic stability of $\mathrm{LaMnO}_{3}$ and its competing oxides: A hybrid density functional study of an alkaline fuel cell catalyst. Phys. Rev. B 2011, 84, 085137.

(15) Symianakis, E.; Malko, D.; Ahmad, E.; Mamede, A.-S.; Paul, J.-F.; Harrison, N.; Kucernak, A. Electrochemical Characterization and Quantified Surface Termination Obtained by Low Energy Ion Scattering and X-ray Photoelectron Spectroscopy of Orthorhombic and Rhombohedral $\mathrm{LaMnO}_{3}$ Powders. The Journal of Physical Chemistry C 2015, 119, 12209-12217.

(16) Mastrikov, Y. A.; Merkle, R.; Heifets, E.; Kotomin, E. A.; Maier, J. Pathways for Oxygen Incorporation in Mixed Conducting Perovskites: A DFT-Based Mechanistic Analysis for (La, Sr) $\mathrm{MnO}_{3-\delta}$. The Journal of Physical Chemistry C 2010, 114, 30173027.

(17) Wang, Y.; Cheng, H.-P. Oxygen Reduction Activity on Perovskite Oxide Surfaces: A Comparative First-Principles Study of $\mathrm{LaMnO}_{3}, \mathrm{LaFeO} 3$, and LaCrO3. The Journal of Physical Chemistry C 2013, 117, 2106-2112.

(18) Ahmad, E. A.; Mallia, G.; Kramer, D.; Kucernak, A. R.; Harrison, N. M. The stability of $\mathrm{LaMnO}_{3}$ surfaces: a hybrid exchange density functional theory study of an alkaline fuel cell catalyst. J. Mater. Chem. A 2013, 1, 11152-11162. 
(19) Bielski, B. H.; Cabelli, D. E.; Arudi, R. L.; Ross, A. B. Reactivity of $\mathrm{HO}_{2} / \mathrm{O}_{2}^{-}$radicals in aqueous solution. Journal of Physical and Chemical Reference Data 1985, 14, 10411100.

(20) Lima, F. H.; Calegaro, M. L.; Ticianelli, E. A. Investigations of the catalytic properties of manganese oxides for the oxygen reduction reaction in alkaline media. Journal of Electroanalytical Chemistry 2006, 590, 152 - 160.

(21) Yeager, E. Electrocatalysts for $\mathrm{O}_{2}$ reduction. Electrochimica Acta 1984, 29, 1527 1537.

(22) Outka, D. A.; Stöhr, J.; Jark, W.; Stevens, P.; Solomon, J.; Madix, R. J. Orientation and bond length of molecular oxygen on $\operatorname{Ag}(110)$ and $\operatorname{Pt}(111)$ : A near-edge x-rayabsorption fine-structure study. Phys. Rev. B 1987, 35, 4119-4122.

(23) Stoerzinger, K. A.; Risch, M.; Suntivich, J.; Lu, W. M.; Zhou, J.; Biegalski, M. D.; Christen, H. M.; Ariando,; Venkatesan, T.; Shao-Horn, Y. Oxygen electrocatalysis on (001)-oriented manganese perovskite films: Mn valency and charge transfer at the nanoscale. Energy Environ. Sci. 2013, 6, 1582-1588.

(24) Scaranto, J.; Mallia, G.; Harrison, N. An efficient method for computing the binding energy of an adsorbed molecule within a periodic approach. The application to vinyl fluoride at rutile $\mathrm{TiO}_{2}(110)$ surface. Computational Materials Science 2011, 50, 2080 $-2086$.

(25) Jensen, F. Introduction to Computational Chemsitry; Wiley, 2007.

(26) Cramer, C. J. Essentials of Computational Chemistry: Theories and Models; Wiley, 2004.

(27) Jia, C.-L.; Lentzen, M.; Urban, K. High-Resolution Transmission Electron Microscopy Using Negative Spherical Aberration. Microscopy and Microanalysis 2004, 10, 174-184. 
(28) Stadelmann, P. A. EMS - a software package for electron diffraction analysis and HREM image simulation in materials science. Ultramicroscopy 1987, 21, 131-145.

(29) Muscat, J.; Wander, A.; Harrison, N. On the prediction of band gaps from hybrid functional theory. Chemical Physics Letters 2001, 342, 397 - 401.

(30) Mallia, G.; Harrison, N. M. Magnetic moment and coupling mechanism of iron-doped rutile TiO2 from first principles. Phys. Rev. B 2007, 75, 165201.

(31) Wilson, N. C.; Russo, S. P.; Muscat, J.; Harrison, N. M. High-pressure phases of $\mathrm{FeTiO}_{3}$ from first principles. Phys. Rev. B 2005, 72, 024110.

(32) Cora, F.; Alfredsson, M.; Mallia, G.; Middlemiss, D. S.; Mackrodt, W. C.; Dovesi, R.; Orlando, R. In Principles and Applications of Density Functional Theory in Inorganic Chemistry II; Kaltsoyannis, N., McGrady, J., Eds.; Structure \& Bonding; Springer Berlin / Heidelberg, 2004; Vol. 113; pp 171-232.

(33) De Fusco, G. C.; Montanari, B.; Harrison, N. M. Half-metallicity in the ferrimagnet Nb(TCNE)2 from first principles. Phys. Rev. B 2010, 82, 220404.

(34) Ge, L.; Jefferson, J. H.; Montanari, B.; Harrison, N. M.; Pettifor, D. G.; Briggs, G. A. D. Effects of Doping on Electronic Structure and Correlations in Carbon Peapods. ACS Nano 2009, 3, 1069-1076.

(35) De Fusco, G. C.; Pisani, L.; Montanari, B.; Harrison, N. M. Density functional study of the magnetic coupling in $\mathrm{V}(\mathrm{TCNE})_{2}$. Phys. Rev. B 2009, 79, 085201.

(36) Liborio, L.; Mallia, G.; Harrison, N. Electronic structure of the $\mathrm{Ti}_{4} \mathrm{O}_{7}$ Magnéli phase. Phys. Rev. B 2009, 79, 245133.

(37) Bailey, C. L.; Liborio, L.; Mallia, G.; Tomić, S.; Harrison, N. M. Defect physics of CuGaS 2 . Phys. Rev. B 2010, 81, 205214. 
(38) Liborio, L. M.; Bailey, C. L.; Mallia, G.; Tomic, S.; Harrison, N. M. Chemistry of defect induced photoluminescence in chalcopyrites: The case of $\mathrm{CuAlS}_{2}$. Journal of Applied Physics 2011, 109, 023519.

(39) Dovesi, R.; Saunders, V. R.; Roetti, C.; Orlando, R.; Zicovich-Wilson, C. M.; Pascale, F.; Civalleri, B.; Doll, K.; Harrison, N.; Bush, I. J. et al. CRYSTAL09 User's Manual. Università di Torino, Torino, 2010.

(40) http://www.crystal.unito.it/Basis_Sets/Ptable.html. 
Graphical TOC Entry

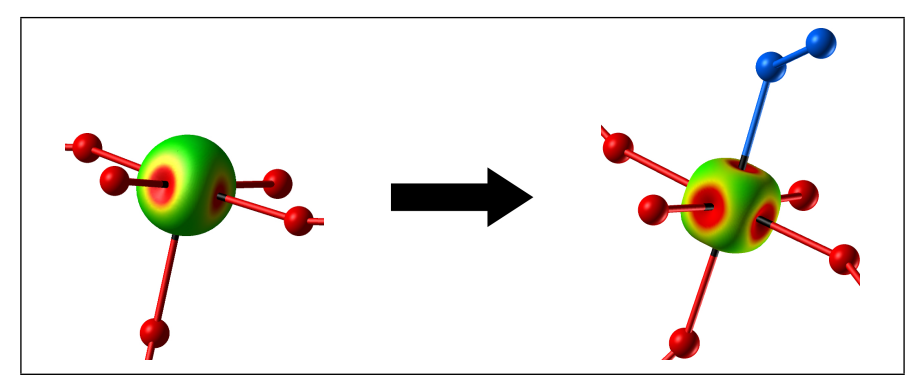

